\title{
Screening for Depression in a Tertiary Epilepsy Clinic
}

\author{
D.W. Gross, M. Davies, J.D. Jirsch, S.N. Ahmed
}

Can J Neurol Sci. 2014; 41: 525-527

\section{RATiONALE}

Symptoms of depression are common in patients with epilepsy with a reported prevalence of as high as $54 \%$. The fact that depression is a strong predictor of quality of life ${ }^{1}$ highlights the importance of developing effective screening tools to assist in the diagnosis of depression in the epilepsy outpatient clinic setting. Gilliam et al have reported that the Neurologic Disorders Depression Inventory in Epilepsy (NDDI-E) is an effective screening tool to detect depression in patients with epilepsy with NDDI-E scores $>15$ associated with a positive predictive value (PPV) of $62 \%$ for major depressive episode (MDE) ${ }^{2}$. Other researchers have also demonstrated high PPV for the NDDI-E ${ }^{3}$, however, the internal reliability of the NDDI-E has not been previously reported. While the replication of validation studies suggests that the NDDI-E is an effective tool to screen for MDE, the results are surprising given the considerable differences in phraseology between the NDDI-E and the Diagnostic and Statistical Manual of Mental Disorders, Fourth Edition, Test Revision (DSM-IV-TR) ${ }^{4}$. Specifically in order to make a DSMIV-TR diagnosis of MDE, a patient must respond affirmatively to experiencing symptoms "most of the day, nearly every day" for five of nine questions while for the NDDI-E a positive score $(>15)$ is possible without any response being in the "always or often" category (i.e. responding "sometimes" to all questions results in an NDDI-E score of 18). The purpose of this study was to evaluate the predictive value of the NDDI-E for MDE in an adult tertiary epilepsy clinic setting. As well, the internal reliability of the NDDI-E was also evaluated.

\section{SubJECTS AND Methods}

Approval was obtained from the University of Alberta Health Research Ethics Board.

Consecutive patients seen in the University of Alberta adult epilepsy clinic were studied. Patients without a diagnosis of epilepsy or with developmental delay were excluded. A total of 100 patients were included in the study (50 female). The mean age of patients was 34 years (range: 16-72). Sixty-one patients were medically intractable.

Patients completed the NDDI-E prior to their outpatient appointment. Demographic data was obtained at the time of their appointments. Patients with NDDI-E scores $>12$ were evaluated further to determine whether the patients had a diagnosis of MDE. Major depressive episode was diagnosed based on direct questioning of patients by epilepsy clinic nurses regarding the presence or absence of the nine DSM-IV-TR criteria for MDE. The PPV was assessed for NDDI-E scores $>12,>15,>17$ and
Table 1: Positive Predictive Value of Different NDDI-E Scores

\begin{tabular}{c|c|c|c}
\hline NDDI-E & $\mathbf{n}$ & MDE & PPV (\%) \\
\hline$>12$ & 57 & 6 & 10 \\
$>15$ & 26 & 6 & 23 \\
$>17$ & 16 & 6 & 38 \\
$>18$ & 11 & 4 & 36 \\
\hline
\end{tabular}

n- number of subjects with NDDI-E scores above the cut-off. MDEnumber of subjects above the cut-off who met DSM IV-TR diagnostic criteria for major depressive episode. PPV- positive predictive value of NDDI-E cut-off for the diagnosis of major depressive episode.

$>18$. Pearson correlation was used to evaluate possible correlations between age and NDDI-E score, with student's t-test used to look for significant differences between NDDI-E score and sex, the presence or absence of seizures and whether patients were taking levetiracetam or topiramate (which have been associated with depression $)^{5}$.

Differences between the responses to the individual NDDI-E items was evaluated using analysis of variance (ANOVA) with Bonferroni corrected Tukey's post hoc pairwise analysis. RELIABILITY: Cronbach's alpha was used to evaluate the overall reliability of the instrument. Monotonicity of individual items was assessed with Pearson's correlation coefficient comparing individual item scores to the rest score (total score omitting the item).

\section{RESULTS}

The mean NDDI-E score was 13.4 (range 6-23). Fifty- seven of 100 patients $(57 \%)$ had a NDDI-E score $>12,26 \%$ had a NDDI-E score $>15,16 \%$ had a NDDI-E score $>17$ and $11 \%$ had

From the Division of Neurology, University of Alberta, Edmonton, Alberta, Canada. Received August 7, 2013. Final Revisions Submitted January 21, 2014. Correspondence to: D.W. Gross, Division of Neurology, University of Alberta, 2E3.19 WC Mackenzie HSC, Edmonton, Alberta, T6G 2B7, Canada.

Email: donald.gross@ualberta.ca. 
Table 2: Item-Rest Score Correlations

\begin{tabular}{|c|c|c|c|}
\hline Question & $\begin{array}{l}\text { Mean } \\
\text { Score }\end{array}$ & $\begin{array}{l}\text { Std } \\
\text { Dev }\end{array}$ & $\begin{array}{c}\text { Item - Rest Score } \\
\text { Correlation }\end{array}$ \\
\hline $\begin{array}{l}\text { Everything is a } \\
\text { struggle }\end{array}$ & 2.47 & 0.83 & 0.67 \\
\hline Nothing I do is right & 2.19 & 0.87 & 0.68 \\
\hline $\begin{array}{c}\text { Feel guilty } \\
\text { I'd be better off }\end{array}$ & 2.23 & 0.93 & 0.56 \\
\hline $\begin{array}{c}\text { dead } \\
\text { Frustrated }\end{array}$ & $\begin{array}{c}1.39^{*} \\
2.97^{* *}\end{array}$ & $\begin{array}{l}0.74 \\
0.70\end{array}$ & $\begin{array}{l}0.45 \\
0.63\end{array}$ \\
\hline $\begin{array}{l}\text { Difficulty finding } \\
\text { pleasure }\end{array}$ & 2.20 & 0.95 & 0.67 \\
\hline
\end{tabular}

Pearson correlation between the individual item and the rest score (total score omitting the individual item). *Significantly lower than other item scores (Bonferroni corrected $\mathrm{p}<0.001$ ). ** Significantly higher than other item scores (Bonferroni corrected $\mathrm{p}<0.001$ ).

a NDDI-E score $>18$ (Table 1). Six patients met DSM IV-TR criteria for MDE all of whom had NDDI-E scores $>17$. The PPV was $10 \%$ for NDDI-E $>12,23 \%$ for NDDI-E $>15,38 \%$ for NDDI-E > 17 and $36 \%$ for NDDI-E $>18$. No correlation was observed between age and NDDI-E score, nor was any sex difference observed (Mean NDDI-E score: males- 13.1, females13.7, $\mathrm{p}=0.4$ ). The mean NDDI-E score was significantly higher in patients with uncontrolled seizures as compared to patients who were seizure free (Mean NDDI-E score: seizure free- 11.9, uncontrolled seizures- 14.4, $\mathrm{p}=0.0006)$. Subjects taking levetiracetam and / or topiramate $(\mathrm{n}=35)$ had significantly higher NDDI-E scores, mean score: 14.5, compared to subjects who were not taking either drug, mean score: $12.8,(\mathrm{p}=0.04)$. While not a direct question on suicide, 13/100 subjects rated their response to item 4 ("I'd be better off dead") as either a 3 or 4 ("always or often" or "sometimes") suggesting potential suicide risk in $13 \%$ of patients. Of note $3 / 13$ subjects whose response to item 4 was $>2$ had a total NDDI-E score $<16$.

Cronbach's alpha was 0.83. Individual item mean scores, standard deviations and Pearson correlations with rest scores are demonstrated in Table 2. Item-rest score correlations ranged from 0.45-0.68. A significant difference between the individual item scores was seen with ANOVA (F-ratio $=65.2, \mathrm{p}<0.001)$ with post hoc analysis demonstrating the responses to item 4 (I'd be better off dead) were significantly lower and responses to item 5 ("frustrated") were significantly higher than the rest of the items (Bonferroni corrected $\mathrm{p}$ values $<0.001$ ).

\section{Conclusions}

Depression is common in epilepsy and has been demonstrated to have a strong correlation with quality of life ${ }^{1}$. The accurate diagnosis of depression in patients with epilepsy is therefore critical in order to achieve optimal patient care ${ }^{2}$. The NDDI-E is an epilepsy specific screening tool that was developed due to concerns that common somatic symptoms often associated with medication side effects (such as fatigue, decreased concentration and sleep disturbance) could affect the specificity of conventional depression screening tools ${ }^{2}$. Previous validation studies have reported favorable results for the NDDI-E with good PPV ${ }^{3}$. We observed high overall NDDI-E scores (mean score 13.4) which were comparable to previous studies (26\% of patients had scores $>15$ in our study compared to $29 \%$ reported by Freidman $e t a l^{3}$ ). In contrast the PPV for the NDDI-E in screening for major depressive episode in our study was much lower (23\%) than the previous validation studies.

There are a variety of possible explanations for the discrepancy between our results and previous studies. Differences between study populations is a possible explanation, however, the similarity in NDDI-E scores between ours and previous reports suggests that this is unlikely. As the NDDI-E scores in our study were comparable to previous studies, the lower PPV must be explained by fewer patients in our study meeting diagnostic criteria for MDE. The most likely explanation for differences in MDE diagnosis relate to the differences in the approach to diagnosing MDE. In our study, MDE was diagnosed based on direct questioning of patients regarding the presence or absence of the nine DSM-IV-TR criteria for MDE by an epilepsy clinic nurse. In contrast the previous validation studies based the diagnosis of MDE on structured clinical interviews with the Gilliam et al and Friedman et al studies both using the Structured Clinical Interview for DSM (SCID) which is a widely used standardized interview designed to be administered by trained clinicians ${ }^{2-3,6}$. A limitation of our study was that the diagnosis of MDE was based on an interview performed by an epilepsy clinic nurse as opposed to a psychiatrist. While it would have been optimal to have a psychiatrist perform the assessment, this was not feasible based on limitation in access to psychiatry (which is consistent with many other tertiary epilepsy centres). While the use of a nurse as opposed to a trained clinician is a possible explanation for the discrepancy between ours and other studies, given the highly structured approach using dichotomous (yes/no) forcedchoice responses to each DSM IV-TR diagnostic criteria, we believe that this is unlikely to explain the differences. The most likely explanation for the discrepancy is differences between the SCID and strict DSM IV-TR diagnosis of MDE. While the SCID is based on the DSM IV-TR, it does not strictly follow the yes/no forced-response format. The approach of the SCID interview is to begin with close ended questions which are followed by a request for elaboration in such a way that if an interviewer suspects that a particular symptom is present he/she does not allow a subject's denial of the symptom to go unchallenged. While the SCID is based on the DSM IV-TR it allows flexibility in arriving at a diagnosis of major depressive episode in contrast to our highly structured dichotomous use of the DSM IV-TR. The difference between the SCID and our highly structured DSM IV-TR based approach is the most likely explanation for the higher incidence of MDE observed in other studies.

Despite the low PPV of the NDDI-E observed in our study, our experience was that the NDDI-E provided clinically important information regarding symptoms of depression. Along with a formal DSM IV-TR based interview, patients with NDDIE scores $>12$ had informal clinic assessments where symptoms of depression and the impact of these symptoms were discussed. 
Although the majority of our patients did not meet a strict DSM IV-TR diagnosis of MDE, most patients with NDDI-E scores $>12$ were experiencing clinically important symptoms of depression. As the importance of subsyndromic depression on epilepsy patients' quality of life is being increasingly recognized, there is a growing trend to treat symptoms of depression when they are disabling regardless of whether a patient meets a strict DSM IV-TR based diagnosis of MDE. Our findings of a Cronbach's alpha of 0.83 and item-rest score correlations ranging from 0.45 to 0.68 support the idea that the NDDI-E has good internal consistency. Given the good internal reliability of the test and our subjective observations regarding the presence of symptoms of depression in patients who did not meet a DSM IVTR diagnosis of MDE, our findings suggest that the NDDI-E is a useful instrument in detecting clinically relevant symptoms of depression in patients with epilepsy. As the focus of previous validation studies has been the diagnosis of major depressive episode, we suggest that further studies are necessary to address the validity of the NDDI-E not just in diagnosing major depressive episode but also in detecting subsyndromic depression.

\section{REFERENCES}

1. Boylan LS, Flint LA, Labovitz DL, Jackson SC, Starner K, Devinsky O. Depression but not seizure frequency predicts quality of life in treatment-resistant epilepsy. Neurology. 2004; 62(2):258-61.

2. Gilliam FG, Barry JJ, Hermann BP, Meador KJ, Vahle V, Kanner AM. Rapid detection of major depression in epilepsy: a multicentre study. Lancet Neurol. 2006;5(5):399-405.

3. Friedman DE, Kung DH, Laowattana S, Kass JS, Hrachovy RA, Levin HS. Identifying depression in epilepsy in a busy clinical setting is enhanced with systematic screening. Seizure. 2009;18 (6):429-33

4. Association AP. Diagnostic and statistical manual of mental disorders 4th ed., text rev. ed. Washington, DC; 2000.

5. Mula M, Sander JW. Negative effects of antiepileptic drugs on mood in patients with epilepsy. Drug Saf. 2007;30(7):555-67.

6. Spitzer RL, Williams JB, Gibbon M, First MB. The Structured Clinical Interview for DSM-III-R (SCID). I: History, rationale, and description. Arch Gen Psychiatry. 1992;49(8):624-9. 